\title{
International Centre for Global Earth Models (ICGEM)
}

\author{
http://icgem.gfz-potsdam.de/home \\ Elmas Sinem Ince, Sven Reißland, Franz Barthelmes (Germany)
}

\section{Overview}

The ICGEM service continues its activities with additional new features introduced in 2017 and 2018. The new features include calculation of the gravity field functionals at user-defined points, a separate collection of the topographic gravity field models, as well as the journal paper which has been published recently as an extensive reference to the ICGEM related activities. ICGEM continues to make the global gravity field models publicly available with a possibility of assigning a DOI number. Although the service was established to collect and provide access to static gravity field models, increasing interest in the temporal gravity field models as well as the topographic and other celestial body gravity field models through the years encouraged the ICGEM team to allocate some effort for archiving and making also these models publicly available. In the following months, new temporal gravity field models developed under the new IAG Service COST-G will be made available via ICGEM. Therefore, some extensions will be applied to the temporal gravity field page.

During the recent years, ICGEM and its products have been used extensively. With the increasing attention to the good scientific practice, copyright and usage restrictions, user inquires regarding referencing the service activities made us reconsider our documentation records. Downloading a model, using a figure published in one of the ICGEM documents or calculation results performed via the calculation service eventually require the user to refer to the service, but our existing reference list was very technical for such purpose. It was not obvious to the reader how to refer to the different activities of the ICGEM. Thinking that a journal paper would be a complementary reference, we prepared an extensive document that is published in Earth System Science Data. Therefore, beside Barthelmes and Koehler 2016 and Barthelmes 2013, the user can now refer to Ince et al., 2019 (https://www.earth-syst-scidata.net/11/647/2019/) for all ICGEM related activities. This paper will also make the ICGEM Service more visible in international platforms.

Finally, the long-time ICGEM service developer and director Franz Barthelmes retired end of 2017. He did invaluable contribution to science and the gravity community. He started and brought such a platform like ICGEM to its current stage. We therefore would like to acknowledge Franz Barthelmes' contributions to gravity field community and GFZ family. The ICGEM will continue its activities with its current two staff given with their time allowance. ICGEM Service can be more informative with the active use of the reshaped discussion forum. The new forum welcomes the users to join the discussion to both ask and answer questions. The forum can be more informative with the contributions from different scientists all over the world.

\section{Activities and publications during the period 2015-2019}

\section{1- The new ICGEM Server}

The ICGEM Service has been renewed from technical, administration and presentation perspectives which was a very important step to develop a new flexible platform for future 
applications and plans also applicable to GRACE-FO mission. The programs used in the calculation service have not been changed. Therefore, the calculations in the new platform are identical to the calculations of previous service settings. Following up the launch of GRACEFO, new products are planned to be made available under the same environment.

The ICGEM Service is actively used for different purposes, e.g. download model, calculate gravity field functionals. The distribution of the visitor statistics of the new service between May 2018 and April 2019 is presented in Fig.1.

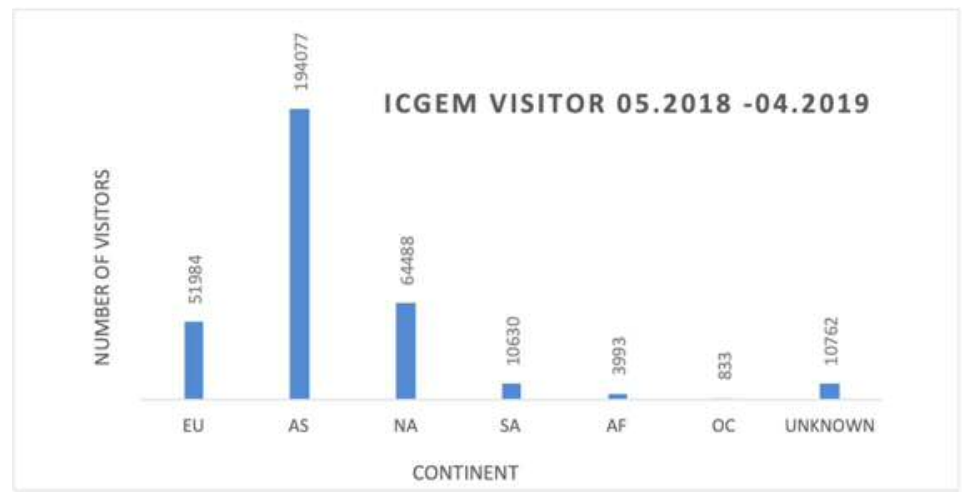

Figure 1: ICGEM Visitor Statistics between May 2018 and April 2019 (EU: Europe, AS: Asia, NA: North America, SA: South America, AF: Africa, OC: Oceania).

As of May 2019, apart from the 169 static gravity field models, we have received Release 6 GRACE models from the three analysing centres and solutions from other groups. The growing interest in the topographical gravity field models also increased the number of the models submitted to ICGEM which is 9 at the moment. Finally, models concerning the other celestial bodies are also of interest to different groups and we received 5 Moon models in 2017 and 2018. Similar to the previous ones, all recently submitted models are provided in the standardised format (Barthelmes and Foerste, 2011) and in the form of spherical harmonic coefficients with possible DOI number assignment via GFZ Library and Information Services (Ince et al., 2019).

The static models (http://icgem.gfz-potsdam.de/tom_longtime), temporal models (http://icgem.gfz-potsdam.de/series) as well topographic gravity field models (http://icgem.gfz-potsdam.de/tom_reltopo) can be found under Gravity Field Models. User can access any reference related to the model that was provided to ICGEM on the same page in column 6 and access to the links to download the model coefficients in column 7, calculate the gravity functionals in column 8 and also to visualise the geoid and gravity anomalies using the link provided in column 9 corresponding the model.

Our evaluations for the static gravity field models are still valid in both spectral domain and wrt GNSS/levelling derived geoid undulations. Spectral comparisons of the models with respect to one of the latest combined models, EIGEN-6C4 can be found under "Spectral domain" (http://icgem.gfz-potsdam.de/evalm). The GNSS/levelling derived geoid undulation comparisons wrt 6 different dataset corresponding to different countries and continents (USA, Canada, Europe, Australia, Japan, and Brazil) are provided in "GNNS/Levelling" (http://icgem.gfz-potsdam.de/tom_gpslev). The columns can be re-ordered by simply clicking on the title of the column. 


\section{2- Calculation of gravity field functionals at user-defined points}

In December 2018, the ICGEM Service introduced the calculation of gravity field functionals at the user-defined list of points. Before, it was only possible to do calculations at grid points that are defined at a spatial resolution of users' request. Now, the user can upload a set of points that are prepared in one of the allowed formats and the gravity field functionals are computed at these points directly. The results are provided on the same page once the computation is finalised.

For the point calculations, after the user uploads the text file of the set of data points in a predefined format (see Fig. 2), the points are displayed on the map. The example in Fig. 2 shows the GNSS/levelling benchmark points in Europe which also are used in the geoid comparisons in the model evaluations. Figure 3 shows the results for the first few points corresponding to the given example in Fig. 2. Different heights for different points can be introduced in the point calculation which is different to the grid calculation where the height is assumed same for all the grid points and consequently delivers results faster. The point calculation was a request from the users, it has been actively used since it has been established.

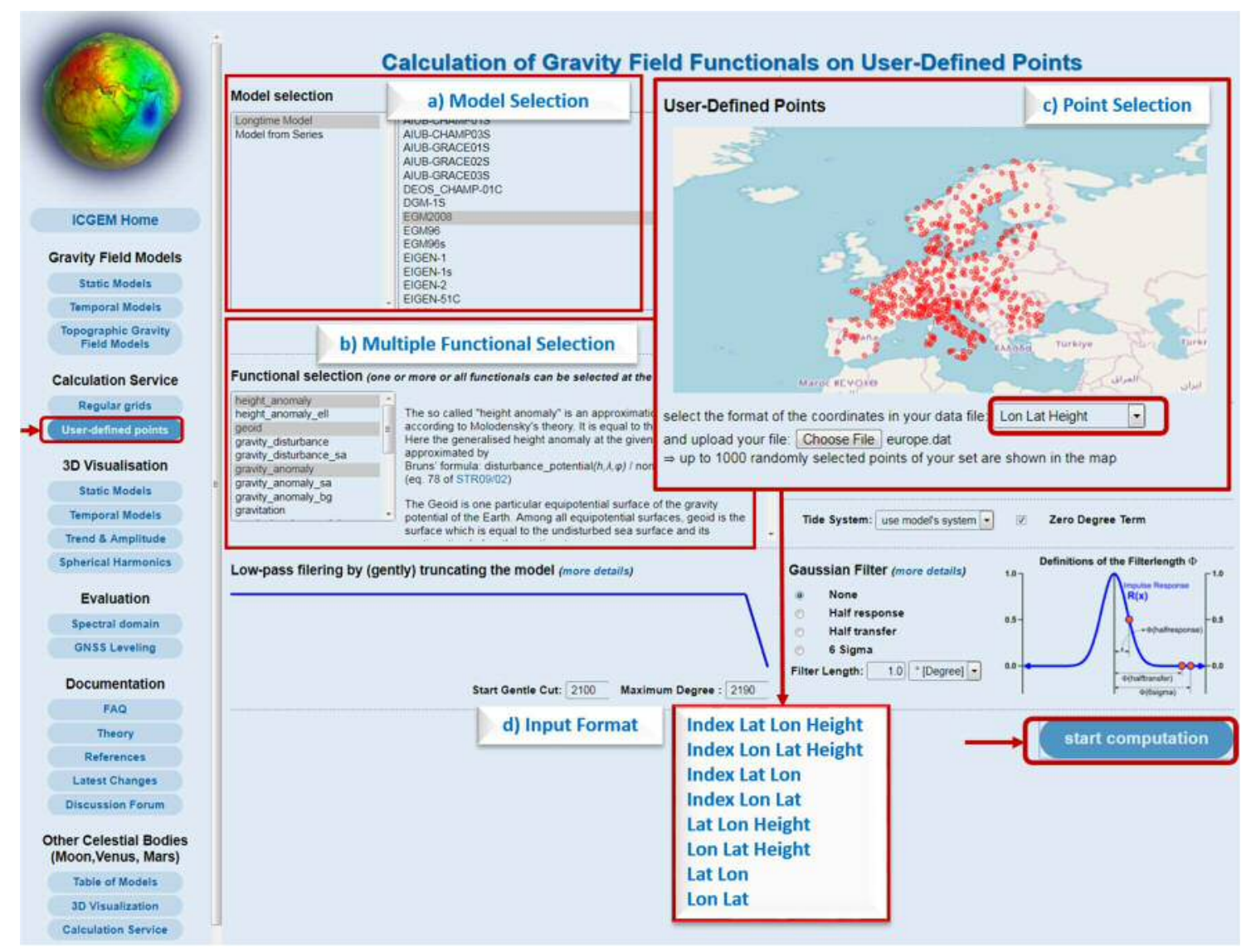

Figure 2: ICGEM Calculation Service - Calculation of Gravity Field Functionals at User-defined Points 


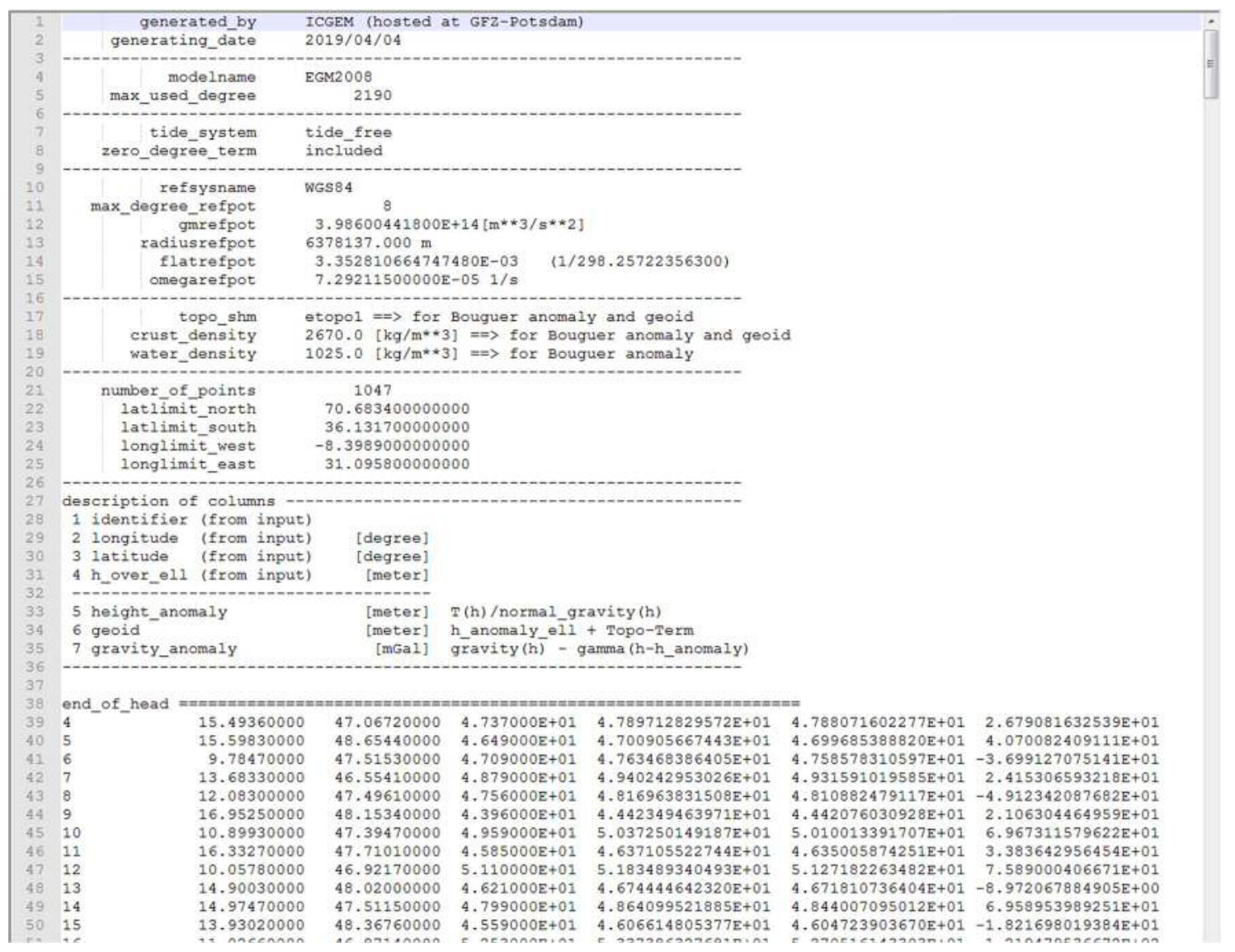

Figure 3: A screenshot of the Calculation Service Point Value Calculation Output as a response to the entry in Figure 2.

\section{3- Documentation}

The new documentation section of the ICGEM Service brings five subsections together to support the scientific community and user interaction. These five subsections are: Frequently asked questions, theory, references, latest changes, and discussion forum. The ICGEM team responds to users' questions as soon as possible in the discussion forum. During the last few years, there were common questions from advanced users, researchers and students that are fundamental to do thorough analyses in different application areas. The ICGEM team has collected frequently asked questions (FAQs) and provided this collection with answers as a pdf document. The questions are answered to meet the needs of both users from different scientific disciplines and experts in the field of physical geodesy. The FAQs list is updated when new questions accumulate. The last version of the FAQs can be accessed via http://icgem.gfz-potsdam.de/faq.

Although the theory of the global gravity field modelling and the calculations of gravity field functionals are technical to be included in this progress report, it is most fundamental to the development of the ICGEM Service. A detailed documentation is reported in Barthelmes, 2013 which includes the potential theory and approximations that are used in the global gravity field modelling.

ICGEM does not only collect gravitational models, but also pays attention on the full documentation of the models. New model releases, new documentation, conference and symposium presentations can be found in the ICGEM Home page and in the list of latest changes. Moreover, for the convenience of the users, all relevant sources are listed in the 
references. This will ensure that the service and its components are available at the same place.

Since the interaction between the users and ICGEM team members involves extensive communications via the service and as well as e-mails, the definition of the guest book was redefined in the last reporting period. The old guest book was modified into a gravity field discussion forum (http://icgem.gfz-potsdam.de/guestbook), which provides users with a platform to communicate with the ICGEM team and other scientists working on similar topics. Apart from fulfilling the requirements of the service, this platform has also been used as a tool for educational purposes in which undergraduate or graduate students communicate with the ICGEM team directly. Anyone without any registration requirement are able to write comments in the forum. However, an approval from the ICGEM team is required in order the comment to be available on the website.

In the following reporting period, this platform should be advertised in the gravity field community to support the ICGEM team and the users. The professionals are also welcome to exchange ideas and answer questions.

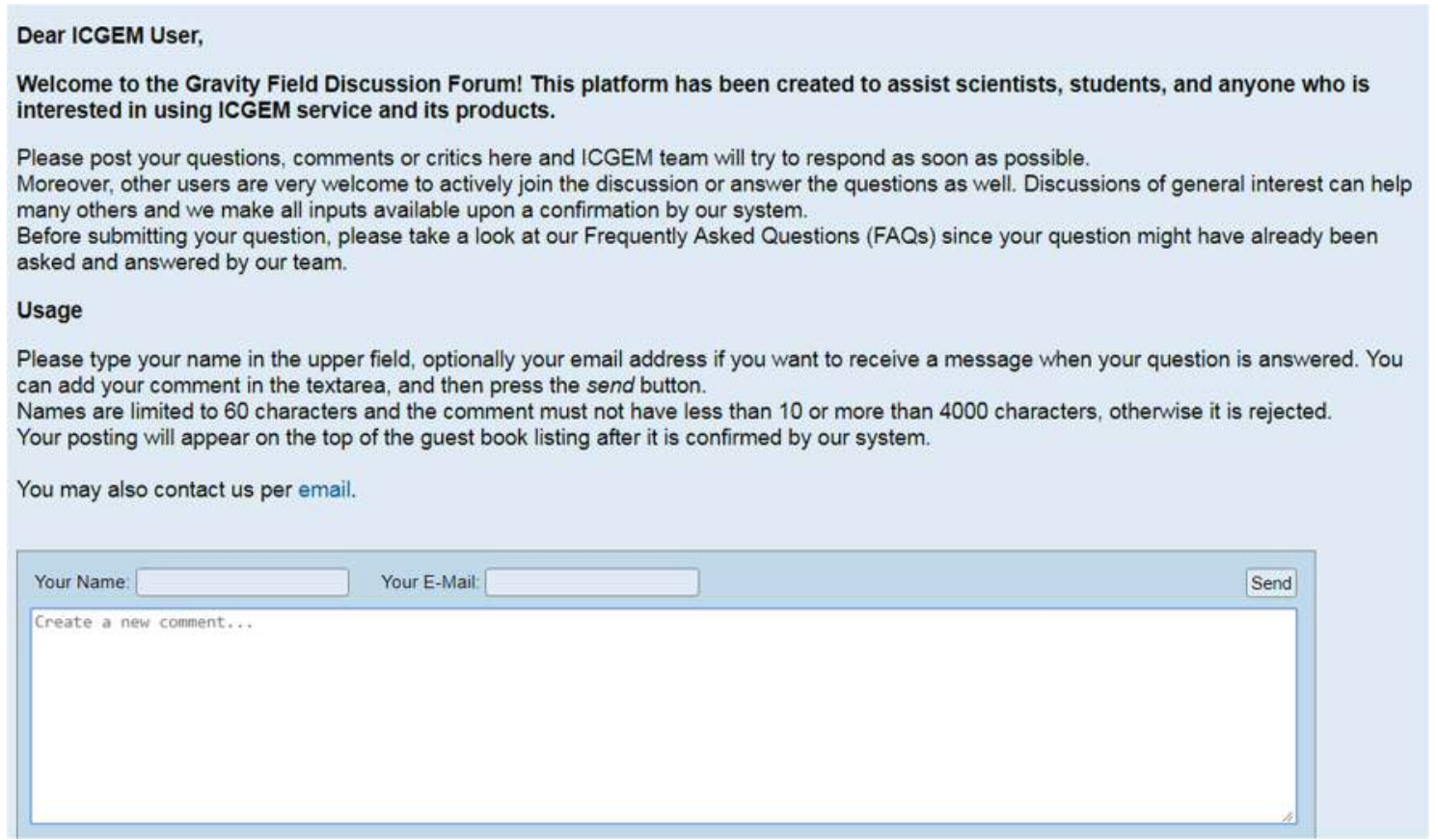

Figure 4: Discussion Forum

\section{4- Presentations and Papers}

Journal paper: Ince, E. S., Barthelmes, F., Reißland, S., Elger, K., Förste, C., Flechtner, F., and Schuh, H.: ICGEM - 15 years of successful collection and distribution of global gravitational models, associated services, and future plans, Earth Syst. Sci. Data, 11, 647-674, https://doi.org/10.5194/essd-11-647-2019, 2019.

EGU poster contribution: New Features and Future Plans of the International Centre for Global Earth Models (ICGEM, http://icgem.gfz-potsdam.de/Ince_et_al_EGU2019_15513_poster.pdf

GGHS2018 poster contribution: New and Long-term Features of the International Centre for Global Earth Models 


\section{Data Policy}

Access to global gravity field models, derived products and tutorials, once offered by the centre, is unrestricted for any external user.

\section{ICGEM Team}

Elmas Sinem Ince (since August 2016)

Sven Reißland (since 2016)

Franz Barthelmes (until December 2017)

\section{Point of Contact}

ICGEM-Team

Helmholtz Centre Potsdam

GFZ German Research Centre for Geosciences

Telegrafenberg, D-14473 Potsdam, Germany

E-mail: icgem@gfz-potsdam.de

\section{References}

Barthelmes, F.: Definition of Functionals of the Geopotential and Their Calculation from Spherical Harmonic Models: Theory and formulas used by the calculation service of the International Centre for Global Earth Models (ICGEM). Scientific Technical Report STR09/02, Revised Edition, January 2013. Deutsches GeoForschungZentrum GFZ, http://doi.org/10.2312/GFZ.b103-0902-26, 2013.

Barthelmes, F., and Förste, C: The ICGEM-format. Potsdam: GFZ German Research Centre for Geosciences, URL: http://icgem.gfz-potsdam.de/ICGEM-Format-2011.pdf, last access 30 January 2019.

Barthelmes, F., and Koehler, W.: International Centre for Global Earth Models (ICGEM). In: Dreves: The Geodesists Handbook 2012, Journal of Geodesy, 86(10): 932-934, https://doi.org/10.1007/s00190-012-0584-1, 2012.

Barthelmes, F., Ince E. S., and Reissland, S.: International Centre for Global Earth Models, International Association of Geodesy, Travaux, Volume 40, Reports 2015-2017, https://iag.dgfi.tum.de/fileadmin/IAG-docs/Travaux_2015-2017.pdf,. 2017, last access 30 January 2019.

Ince, E. S., Barthelmes, F., Reißland, S., Elger, K., Förste, C., Flechtner, F., and Schuh, H.: ICGEM - 15 years of successful collection and distribution of global gravitational models, associated services, and future plans, Earth Syst. Sci. Data, 11, 647-674, https://doi.org/10.5194/essd-11-647-2019, 2019. 